\title{
Prends soin du métro. Le contrôle-soin, forme émergente des régulations et menaces souterraines
}

\author{
Cuide do metrô. O controle-cuidado, forma emergente dos regulamentos e \\ ameaças subterrâneas
Take care of the metro. The care-control, emerging form of underground regulations and threats

Yves Jouffe ${ }^{[a]}$, Diego Carvajal[b] Alejandra Lazo $^{[c]}$

\author{
[a] Université Paris-Est, Paris, France \\ [b] Universidad de Artes y Ciencias Sociales (UARCIS), Santiago, Chili \\ [c] Universidad de Los Lagos, Santiago, Chili
}

\section{Résumé}

Le métro de Santiago a subi une dégradation significative de ses conditions de transport du fait de son intégration au Transantiago, le réseau de bus urbains. L'opérateur du métro a donné beaucoup de visibilité à ses dispositifs de contrôle des nouvelles masses d'usagers : barrières optimisées, signalétique omniprésente, agents démultipliés, etc. L'étude du discours officiel de l'entreprise et surtout l'observation ethnographique de ces dispositifs révèlent que les modalités de contrôle ont elles-mêmes évoluées. En plus de la menace légale exercée contre les étrangers et de la régulation des flux par une discipline des corps, un contrôle-soin s'exerce sur une population à protéger : la population qui bénéficie du soin de l'opérateur lui est redevable d'un contre-soin. Le contrôle-soin constitue non seulement une modalité émergente de contrôle mais aussi la forme générale du contrôle dans le métro de Santiago.

Mots-clés: Contrôle. Soin. Mobilité quotidienne. Métro. Santiago du Chili.

\section{Resumo}

O metrô de Santiago sofre com uma degradação significativa de suas condições de transporte pelo fato de ser integrado ao Transantiago, sistema de ônibus urbanos. A empresa operadora do metrô colocou em prática dispositivos de controle com o objetivo de monitorar a nova massa de usuários: roletas otimizadas, sinais, agentes de controle, etc. Desse modo, e a partir do estudo do dispositivo oficial da empresa operadora e, sobretudo, da observação etnográfica de tais dispositivos, observamos uma evolução dos sistemas de controle. Além da ameaça legal exercida contra os estrangeiros e a regulação do fluxo pela disciplina do corpo através de um controle-cuidado exercido pela operadora sobre a população a ser protegida: a população que se beneficia do cuidado da operadora é obrigada a retribuir com um contracuidado. Tal contracuidado se constitui não somente da modalidade emergente de controle, mas também de uma formal geral do controle no metrô de Santiago.

Palavras-chave: Controle. Cuidado. Mobilidade cotidiana. Metro. Santiago do Chile.

YJ est Sociologue, Dr de l’Ecole des Ponts ParisTech, e-mail: yves.jouffe@gmail.com DC est Sociologue, Licencié de l’Universidad ARCIS, e-mail: diegocarvajalhicks@gmail.com AL est Anthropologue, Dre en Géographie de l'Université Toulouse Le Mirail et P. Universidad Católica de Chile, e-mail: alejandra.lazo@ulagos.cl 


\section{Abstract}

The Underground System of Santiago, Chile has undergone significant deterioration of its transportation conditions since it was integrated to the 'Transantiago' - the metropolitan bus network. The Underground operating company has given a great deal of visibility to the control devices of its new masses of users: optimized turnstiles, ubiquitous signage, increased number of agents, etc. The study of the operating company's official speech and, above all, the ethnographic study of these devices show that the modes of control, themselves, have evolved. On top of the legal threat to foreigners and the regulation of flow through a discipline of the body, a care-control is being conducted on the population that requires protection: the people who are being cared for by the operating company are required, in return, to care for the Underground. The care-control not only constitutes an emerging mode of control, but it is also the general form of control in the Santiago's Underground.

Keywords: Control. Care. Daily commuting. Underground. Santiago, Chile.

\section{Introduction}

L'extension du métro de Santiago du Chili vers les banlieues pauvres et la mise en place en février 2007 du Transantiago, le système de bus rapides, se sont traduites par un afflux massif de nouveaux usagers dans le métro (Díaz et al., 2006). Ses conditions d'utilisation se sont fortement dégradées en certains endroits et certaines heures, la concentration humaine atteignant jusqu'à plus de 6 personnes par $\mathrm{m}^{2}$ et suscitant des conflits d'usage inédits (Ureta, 2013). Certaines stations de correspondances se sont vues complètement congestionnées aux heures de pointe, les files d'attente géantes envahissant les couloirs d'accès aux quais (Lazo, 2008).

L'entreprise Metro, opérateur gestionnaire du métro de Santiago, avait anticipé la congestion et les conflits en résultant. Cependant, ses responsables pointaient avant tout l'inadaptation culturelle des populations pauvres amenées à utiliser en masse le métro auparavant réservé aux populations riches du centre, dotées, elles, d'une « culture métro » (Ureta, 2013). Ureta qualifie ce discours comme un rejet des « barbares ». Ce dénigrement réactive en effet un imaginaire collectif sur Santiago, lequel oppose « ville civilisée » et « ville barbare» (Márquez, 2012). La « culture métro » serait attachée à certaines personnes qui jouiraient d'un accès exclusif à ce territoire souterrain.
Les nouveaux usagers y apparaissent inadaptés parce que pauvres et banlieusards, c'est-à-dire comme des étrangers, à exclure. Cette exclusion est la fonction des vigiles, barrières et autres panneaux menaçants du métro. Des responsables de Metro évoquent aussi «l'effet métro » (Ureta, 2013) qui désignerait la capacité de l'espace même du métro à imposer à chacun les bons comportements, par exemple au travers des ordres des assistants de quai. Les usagers ainsi disciplinés sont assimilés dans une classe «moyennisée » par le système de mobilité urbaine lui-même (Jouffe, 2015). Ces deux modalités de contrôle, interdiction des « barbares » et imposition du bon comportement, correspondent à certains dispositifs du métro. Mais d'autres modalités peuvent exister et se développer alors que l'expérience du déplacement souterrain est bouleversée par les masses du Transantiago et par les nouveaux dispositifs qui les prennent en charge.

Nous proposons de caractériser l'articulation des différentes modalités de contrôle des usagers en nous focalisant sur les nombreux dispositifs matériels qui visent à influencer leurs comportements. Après avoir défini comme modalités de contrôle la menace, la régulation et le soin, nous les identifierons dans les dispositifs du métro à partir d'une observation ethnographique. Enfin, nous les articulerons entre elles afin de reconnaître non seulement la nouveauté mais la prépondérance du contrôle-soin sur les régulations et menaces du transport urbain souterrain de Santiago du Chili. 


\section{Menace, régulation, soin}

\section{Articulation des mécanismes de souveraineté}

La présence oppressante des corps et la tentative de leur maîtrise technologique constituent une dimension prégnante de l'expérience commune du métro et en font l'espace clos typique des sociétés disciplinaires (Deleuze, 1990). Les dispositifs de régulation des flux (architecture intérieure, signalétique, assistants de quai, etc.) imposent à chaque corps le même comportement défini pour optimiser le flux et la sûreté. Ils correspondent à la « discipline» comme conduite normée des conduites (Foucault, 1975). En plus de cette discipline, nous défendrons l'idée de la permanence d'une menace d'ordre « légal » et de l'émergence d'un soin d'ordre « biopolitique ». D’une part, des dispositifs de menace (gardiens, barrières, etc.) visent à exclure les personnes ne souhaitant pas voyager ou payer pour le faire. Ils appliquent une loi souveraine qui se fonde sur l'interdiction de certains comportements sur un territoire et sur la séparation entre les sujets soumis à la loi et les "étrangers » susceptibles d'expulsion (Foucault, 2004a). D'autre part, nous souhaitons montrer que l'espace clos du métro n'est pas que disciplinaire ou soumis à la loi mais qu'il se laisse remplir par l'espace continu des « sociétés de contrôle» (Deleuze, 1990). De nouveaux dispositifs (signalétique incitative, agents de service au client, etc.) affirment prendre soin des usagers dans leur diversité. Ils correspondent à une technologie « biopolitique » de gouvernement, c'est-à-dire qui s'appuie sur la diversité de la population pour inciter plutôt qu'obliger ou interdire ses conduites (Foucault, 2004b).

Nous insisterons sur la coexistence et la combinaison des modalités de contrôle, sous la forme dominante du soin. En effet, comme le rappelle Edgardo Castro (2008, note 8), Foucault (2004a, p. 7-9) précise explicitement qu'il n'y a pas une époque de la souveraineté, puis une autre des disciplines et enfin une autre de la biopolitique, mais que les trois mécanismes de la souveraineté (légaux, disciplinaires et biopolitiques) coexistent et l'un se voit plus accentué que les autres pendant certaines époques. De même, tous les éléments du métro agissent ensemble sur les usagers avec divers principes d'action. C'est même l'action synchronique d'éléments hétérogènes qui garantit leur efficacité. Ces éléments eux-mêmes sont combinés et hybrides, comme une télévision diffusant de la musique qui construit une ambiance avec une décoration et contre des odeurs contingentes, des informations sur l'actualité hors métro faisant écho aux journaux quotidiens gratuits, de la publicité qui se bat pour voler à l'affichage publicitaire les instants d'attention des usagers, et des consignes de sécurité redondantes avec celles affichées sur les quais.

Les trois mécanismes de souveraineté distingués par Foucault décrivent des modes de structuration du pouvoir à l'échelle de la société. Ils résultent de l'action complexe d'innombrables dispositifs concrets qu'il peut être difficile d'associer à un mécanisme plutôt qu'à un autre. Nous allons donc spécifier ces mécanismes en trois modalités situées de contrôle (menace, régulation, soin) afin de les reconnaître dans les dispositifs concrets du métro. Nous allons en particulier enrichir le concept de soin d'autres apports théoriques.

\section{Le contrôle-soin matriarcal}

Nous proposons de tirer parti de l'idée de contrôle matriarcal pour spécifier le soin comme type de contrôle. L'éthique du soin ne se décline pas directement en une modalité de contrôle. Toutefois, les théories féministes du "care » critiquent à la fois l'hégémonie masculine de l'éthique de la justice et la féminisation de l'éthique du soin qui participe de la marginalisation réciproque des femmes et du soin (Cova, 2010). Les stéréotypes du soin féminin dessinent alors une modalité de contrôle matriarcal, susceptible de nourrir un mécanisme biopolitique de normalisation.

Ce contrôle matriarcal s'inscrit dans des types métaphoriques de relations familiales de contrôle qui s'inspirent des trois mécanismes de souveraineté déjà mentionnés et des cosmovisions classiques selon Ortiz Osés (2001) : droit patriarcal de punition sur son territoire à fin de soumission (contrôle-menace légale), savoir «fratriarcal» de surveillance mutuelle partout où vont les frères $\mathrm{y}$ compris soi-même à fin d'apprentissage (contrôlerégulation disciplinaire), et créance matriarcale 
de protection de la mère en son foyer à fin de consentement (contrôle-soin biopolitique). Les types de relations de pouvoir existant entre une masse innombrable d'usagers et une institution pyramidale et médiatisée par de nombreux dispositifs techniques ne sauraient se réduire à ceux existant au sein d'une famille. Le recours à cette typologie semble pourtant pertinent car nous retrouvons dans les discours de Metro et les dispositifs du métro des références à cette éthique du soin et de la responsabilité et à ces ordres familiaux, en particulier à la créance matriarcale.

Le fondement du pouvoir matriarcal est la dette absolue vis-à-vis de la personne qui a donné la vie («fais ce que je te dis car tu me dois tout»), ou la soumission à la personne qui veut du bien («fais ce que je te dis car c'est pour ton bien »). Le contrôle-soin matriarcal serait un contrôle qui s'exerce au travers du soin donné ou annoncé, soin qui s'appuie sur du contrôle donc le justifie et en produit les conditions. Le contrôle-soin n'est donc pas la simple concomitance du contrôle et du soin. Le contrôle-soin donné impose un contre-soin tout comme le don impose un contre-don; mais alors que contre-dons et dons s`enchaînent et se surpassent respectivement en créant une dette mutuelle, le contre-soin ne peut compenser le contrôle-soin, radicalement asymétrique comme lest le don maternel de la vie. Une claire illustration dans le cas du métro de Santiago en est l'injonction publicitaire «Prends soin du métro » (« Cuida al metro ») associée à l'affirmation « Le métro prends soin de toi » («El metro te cuida»). Le métro exige une soumission fondée dans le soin même donné à l'usager. Il ne s'agit pas d'une réciprocité comme la symétrie de la publicité pourrait le faire croire, car le soin est asymétrique comme dans la relation matriarcale : le métro protège la vie de l'usager et l'usager doit respecter les consignes dictées par le métro.

Les trois modalités de contrôle seront finalement définies comme suit :

- la menace vise l'interdiction de la fraude, des agressions et des dégradations au titre de la menace envers l'opérateur ;

- la régulation vise l'obligation du placement des corps ajusté au flux au titre du maintien du fonctionnement ;
- le soin vise l'incitation d'un comportement bénéfique à l'usager et qui fonde son contrôle au titre du développement de la diverse population.

\section{Méthode}

L'analyse des dispositifs de contrôle mobilise principalement un travail ethnographique d'observation participante (Velasco Maillo \& Díaz de Rada, 1997, p. 93), d'abord flottante (Pétonnet, 1982) puis focalisée sur l'interaction entre corps et dispositifs matériels, humains et non-humains déployés par Metro, dans l'enceinte du métro de Santiago du Chili. Celle-ci comporte les voitures, quais, couloirs, zones de contrôles, espaces souterrains de consommation et entrées publiques. L'observation réalisée dans d'autres espaces du transport urbain notamment les bus et les gares multimodales ne sera pas mobilisée ici. Pendant les mois de mars et avril 2009, les parcours ont été quotidiens et systématisés de façon à intégrer l'ensemble des lignes et stations et la diversité des horaires et jours de service. Des observations moins fréquentes mais aussi attentives ont précédé cette période intense de quelques mois et surtout l'ont prolongée en 2009 et 2010 en complément d'un travail d'analyse, puis de manière récurrente mais ponctuelle, pendant plusieurs années jusqu'à aujourd'hui en 2014. Ce travail s'appuie sur le remplissage d'observations et d'impressions dans un carnet de terrain en tant que voyageur percevant, éprouvant et réalisant à la fois la performance des dispositifs de contrôle du métro au travers de l'expérience située du déplacement. Des entretiens improvisés et annotés ont été aussi réalisés avec les différents types d'agents en situation de travail et non de déplacement dans l'enceinte du métro, des vigiles aux vendeurs, ainsi qu'avec des voyageurs. Des entretiens plus formels ont été réalisés en face à face avec quelques responsables de stations et, au moyen de messages électroniques, avec la responsable de la communication de l'entreprise gestionnaire, Metro. L'analyse des documents officiels émis par le service de communication, rapports annuels ${ }^{1} 2009$ et animation du compte

\footnotetext{
${ }^{1}$ Rapports téléchargeables sur www.metrosantiago.cl
} 
Twitter et Facebook de Metro, complète le dispositif méthodologique.

\section{L'articulation des contrôles en dispositifs}

\section{Contrôle signalétique par le soin}

La signalétique constitue un des dispositifs les plus visibles parmi ceux mis en place pour recevoir et éduquer les nouveaux usagers du Transantiago. Comme le montre notamment Ureta (2013), elle a rempli l'espace intérieur du métro : rames, quais, couloirs, entrées de stations, sur les vitres, les panneaux, les murs, les sols et les tranchées de circulation des rames. Cette signalétique est essentiellement structurée par le contrôle-soin, même si son contenu renvoie en grande partie à la régulation et à la menace.

La campagne de promotion «Le métro prend soin de toi » ( "El metro te cuida») évoluera en un message double (évoqué plus haut) «Le métro prend soin de toi. Prends soin du métro » qui révèle comment la prétention de soin cache (à peine) une injonction et revendique un contrôle sur l'usager. Ce contrôle se formule comme une exigence de contre-soin en retour. Le contrôle-soin est ici entier.

L'autre campagne de promotion du métro avait pour titre « Te llevo bajo la piel » qui se fonde sur un jeu de mots entre "Je t'ai dans la peau » et " Je te transporte sous la surface de la terre ». Une identité est donc proposée entre la justification essentielle du métro comme moyen de transport et une relation à la fois corporelle et affective. La relation affective renvoie explicitement à l'attachement que le soin produit, notamment dans sa formulation comme "care », et qui fonde son effectivité comme contrôle. Nous proposons d'interpréter la dimension corporelle comme une reconnaissance et une revendication implicites de la nature globalement disciplinaire du métro.

Une autre campagne s'intitulait " $M i$ Metro. Tu Metro ( ( Mon métro, ton métro »). Ce slogan renvoie, implicitement mais clairement, à un bien partagé, qui implique une responsabilité distribuée du soin. C'est bien le principe de responsabilité que le " care » attache au soin. Nous retrouvons l'idée formulée par les concepteurs, agents et usagers : le métro d'avant le Transantiago se caractérisait par un soin entre passagers, aujourd'hui contredit par la lutte sans merci que se livrent les usagers pour leurs places (Ureta, 2013). Ce devoir de soin active la relation d'ordre familial ou communautaire (peut-être 《 fratriarcal ») contre une responsabilité déléguée par exemple à des préposés au nettoyage ou à la surveillance. Cette lecture familiale est également directement mobilisée dans une campagne ciblée de promotion de nouvelles rames de métro, sur lesquelles sont collées des affiches géantes : « 36 nouveaux membres dans la famille» ( 36 nuevos integrantes de la familia »). La famille apparaît ici comme modèle de relation à la fois avec les machines du métro et avec la communauté des usagers. Cette famille appelle une solidarité c'est-à-dire un soin mutuel en même temps qu'un contrôle mutuel de ce soin. Elle suggère aussi l'existence d'une autorité supérieure aux machines et aux usagers, c'est-à-dire, logiquement dans cette enceinte, l'opérateur Metro.

Cet appel récurrent au soin des usagers envers le métro renvoie implicitement à son interprétation littérale pour un soin accordé à un objet, c'est-à-dire sa non-dégradation par salissure ou vandalisme, ce que renforcent de nombreux autres éléments signalétiques. Mais il se lit aussi en termes de respect du mode d'emploi de l'objet, c'est-à-dire de l'ensemble des règles énoncées par Metro, dont beaucoup visent un contrôle-régulation et un contrôle-menace.

L'essentiel des éléments signalétiques s'inscrivent dans une « campagne d'habitudes » (« campaña de hábitos ») ainsi nommée par l'opérateur. Elle étend des messages contextuels sur l'ensemble des surfaces du métro, les désignant ainsi toutes comme des supports impliquant une utilisation adaptée par les usagers. Chaque message combine un appel générique au soin du métro ( «Por un Metro amable/seguro/limpio/solidario» : «Pour un métro convivial/sûr/propre/solidaire ») et une consigne (message et icône) adaptée à sa localisation précise (Figure 1). Cette consigne est de diverse nature :

- régulation des flux («PORUN METRO AMABLE: Circula siempre por tu derecha en andenes $y$ pasillos »: « Pour un métro convivial : circule toujours sur ta droite sur les quais et dans les couloirs »),

- régulation des flux et confort de l'usager ( AVANZA HACIA LOS EXTREMOS DEL ANDEN: Estarás más cómodo »: "Avance vers les extrémités du quai : tu seras plus à l'aise »)

- régulation des flux et intérêt de l'usager pressé ( « POR UN VIAJE EXPEDITO: No impidas el cierre 
de las puertas » : «Pour un voyage rapide: n'empêche pas la fermeture des portes »; « POR UN VIAJE EXPEDITO: Deja bajar antes de subir » : «Pour un voyage rapide : laisse descendre avant de monter »)

- $\quad$ protection des usagers et régulation des flux (« POR UN VIAJE SEGURO: Traspase la línea amarilla solo para subir al tren »: " Pour un trajet sûr : ne franchissez la ligne jaune que pour monter dans le train »; et sa variante: «POR SU SEGURIDAD: Traspase la línea amarilla solo para abordar al tren » : «Pour votre sécurité : ne franchissez la ligne jaune que pour entrer dans le train »),

- protection des usagers ( POR UN VIAJE SEGURO: No te apoyes en la puerta » : «Pour un voyage sûr : ne t'appuie pas sur la porte»; également sept consignes affichées près des escalators de chaque station)

- soin entre usagers ( $\quad P O R$ UN METRO SOLIDARIO: Cuida a los niños, embarazadas y ancianos » : «Pour un métro solidaire : prends soin des enfants, des femmes enceintes et des personnes âgées »)

- $\quad$ protection du métro par les usagers vis-à-vis des autres (les taggueurs délinquants) plutôt que vis-à-vis d`eux-mêmes (lorsquiils salissent le métro) ("Sigamos ayudando a que no rayen el Metro. El Metro te cuida, cuida el Metro. LLAMA $A L 800540800$ » : « Continuons d'aider à ce qu'ils ne mettent pas de graffiti sur le métro. Le métro prend soin de toi, prends soi du métro. Appelle le 800540 800. »; « POR UN METRO AMABLE: Ayuda a mantenerlo limpio » : « Pour un métro convivial : aide à le maintenir propre »)

Ces consignes visent les trois fonctions de régulation des flux (y compris dans la disposition des masses d`usagers sur les quais), de soin des usagers (confort et protection de leur intégrité corporelle) et de menace contre les agressions (souillure des équipements). Mais quelle que soit la fonction visée, la consigne met en avant le soin accordé aux usagers. Cette campagne signalétique affiche et affirme ainsi la légitimité supérieure du contrôle-soin sur les autres modalités de contrôle.

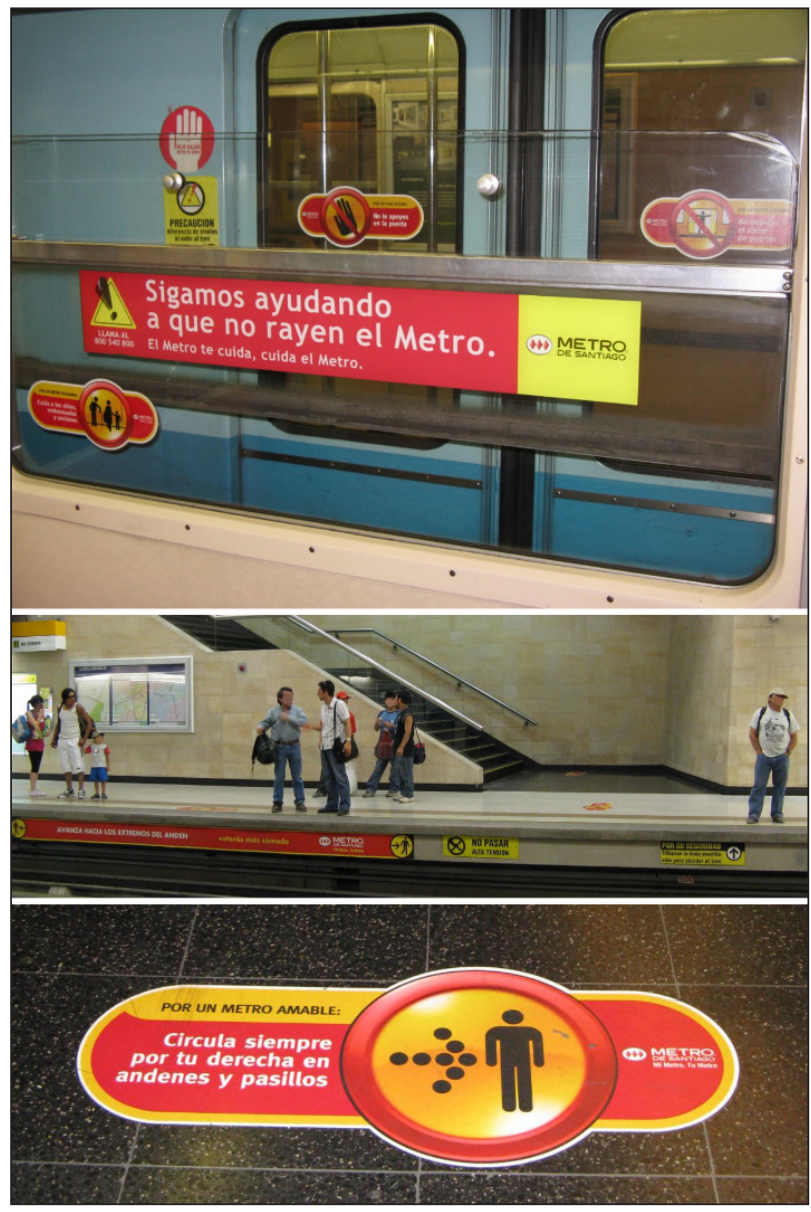

Figure 1 - « Campagne d'habitudes 》, métro de Santiago du Chili, 2009 Source: photos des auteurs.

Mélange inédit des modalités de contrôle

Très peu d'éléments signalétiques ne font pas référence au soin bien que leur taille et leur fonction régulatrice les associent à la même " campagne d'habitudes ».

De même, d'autres consignes régulatrices largement diffusées (de chaque côté de chaque porte des rames de métro) n'ont pas d'introduction par le soin ( «DEJE BAJAR ANTES DE SUBIR » : « Laissez descendre avant de monter »), mais se trouvent toujours associées à des consignes de protection ( $\ll$ PRECAUCION diferencia de niveles al subir al tren » : « Attention : différence de niveau entre le sol et le métro »).

D'autres signalétiques plus discrètes (plus petites, moins colorées et plus simplement composées, parfois sans texte ou sans icône) et sans slogan orienté vers le soin appartiennent certainement à une génération plus 
ancienne. Elles sont orientées vers la sécurité corporelle des voyageurs et prennent la forme dinterdictions sèches, de l>ordre du contrôle-menace ( NO ENCENDER FUEGO. NO FUMAR» : « Ne pas allumer de flamme. Ne pas fumer »; «NO PASAR / ALTA TENSION» : « Ne pas passer/ Haute tension »).

La comparaison entre les deux générations de signalétiques nous fait mettre l'accent sur la nouveauté de certaines caractéristiques : la diversification des consignes et des fonctions, la cohérence graphique, la visibilité, la lisibilité, le tutoiement voire le «jejoiement» (Amorim, 2015) (au lieu du vouvoiement ou de l'infinitif), l'association systématique à un slogan dont le qualificatif appelle au soin des usagers pour leur métro même lorsque la consigne ne renvoie pas directement au soin. Contre la froide formalité, le tutoiement renvoie encore aux relations affectives typiques de la famille dont les membres se connaissent et prennent soin les uns des autres.

Le mélange des modalités de contrôle entre consigne et slogan et entre consignes nous fait supposer qu'elles se renforcent mutuellement dans la mesure où les usagers ne sont pas a priori sensibles au même type de contrôle et où l'efficacité d'une consigne gagnera les autres consignes similaires, surtout lorsqu'elles sont intégrées dans un même élément signalétique ou dans une même campagne (même charte graphique et déploiement synchrone).

\section{Premier contrôle-soin: exiger le soin aux plus fragiles}

Plusieurs éléments signalétiques, ou déployés dans le cadre d'une campagne signalétique, nous semblent plutôt associés à la régulation disciplinaire des corps. Le plus explicitement disciplinaire est la campagne du «passager incognito », réalisée en 2008, en même temps que la « campagne d'habitudes ». Le « passager incognito » est une célébrité qui suit incognito des passagers et qui offre une prime (une trentaine de voyages sous la forme d'une carte « bip » chargée avec 15.000 pesos chiliens) à dix usagers par jour, pour récompenser l'intégration des bonnes habitudes. La récompense de l'incorporation du bon comportement participe ici de l'obligation disciplinaire, confirmée par une observation secrète qui vise à incarner un contrôle panoptique.
Cette campagne s'est appuyée sur un « protocole de bonnes habitudes de voyage $»^{2}$, rendu public à cette occasion et censé correspondre aux règles essentielles à suivre dans le métro. Ces consignes correspondent aux anciennes signalétiques tant sur la forme, infinitive et sobre, que sur le fond, pour une régulation non justifiée par le soin. L'exception est l'exigence de soin à accorder aux femmes enceintes, aux personnes handicapées et aux personnes du troisième âge (consigne $\mathrm{n}^{\circ} 7$ ). Ce soin aux personnes fragiles se retrouve seul au milieu de neuf consignes de régulation des mouvements, soit pour l'optimisation des flux $\left(\mathrm{n}^{\circ} 1,2,4,8,9\right)$, soit pour l'intégrité corporelle des usagers $\left(\mathrm{n}^{\circ} 3,5,6,10\right)$, dont la protection optimise aussi les flux en évitant les interruptions de trafic pour accident de voyageur. On retrouve cette configuration dans un autre dispositif signalétique, à savoir les sept consignes affichées à côté de chaque escalator : une consigne de soin aux plus fragiles parmi des consignes de régulation pour la protection des usagers. La consigne de soin des plus fragiles ne relève pas exactement de la régulation disciplinaire. Elle est d'un autre ordre, biopolitique, car elle reconnaît et préserve la normalité d'une sous-population d'usagers.

Le soin aux personnes fragiles apparaît comme le premier élément de contrôle-soin à s'inscrire dans une signalétique de régulation disciplinaire. Il ne

${ }^{2}$ La campagne « Passager incognito » a eu lieu du 8 juillet au 2 septembre 2009 et a visé l'inculcation des comportements suivants:

«Protocolo de buenos hábitos de viaje

1) Dejar bajar antes de subir al tren

2) No entorpecer el cierre de puertas del tren

3) Cruzar la línea amarilla instalada en los andenes sólo para subir al tren

4) No ingresar al tren al encenderse la luz roja de cierre de puertas

5) No apoyar las manos en las puertas del tren

6) Prevenir caídas al interior del tren usando pasamanos y manillas

7) Ceder el asiento a embarazadas, minusválidos y personas de tercera edad

8) No circular en contra del flujo de pasajeros en escaleras y pasillos

9) Ubicarse al lado derecho de las escaleras mecánicas en caso de no ir apurado

10) No correr en pasillos, escaleras y andenes »

Source: Metro Santiago, "Pasajero Incógnito: 220 usuarios de Metro han sido premiados por sus buenas conductas en el tren subterráneo", Noticias, Lunes 10 de Agosto 2009, URL: http:// www.metro.cl/prensa/detalle/979d472a84804b9f647bc18 5a877a8b5, consulté le 4 mars 2015. 
s'agit pas d'un contre-soin au sens strict, à savoir un soin de l'usager pour Metro au titre du soin de Metro pour l'usager. En effet, Metro exige un soin des usagers normalement capables pour les autres usagers au titre d'un principe moral supérieur. Toutefois ce soin aux plus fragiles s'approche du contre-soin et le préfigure. L'entreprise Metro communique dans ses rapports publics sur ses propres opérations d'aménagement en faveur de «l'accessibilité », qui désigne en fait celle des " personnes à mobilité réduite » (PMR). La consigne peut alors se lire comme contrôle-soin c'est-à-dire exigence de contre-soin : Metro prend soin de la population générale des usagers en se préoccupant des plus fragiles d'entre eux, ce qui l'amène à exiger en retour, de la part de tous les usagers, un contre-soin qui aille dans le même sens que Metro, à savoir le soin des plus fragiles. Ce contrôle-soin pour les plus fragiles initie le mélange des modalités de contrôle sans que le contrôle-soin n'en soit encore la forme privilégiée. La légitimité de cette exigence de soin bénéficie déjà aux consignes de régulation voire de menace par association analogique. Elle annonce surtout, voire prépare, la généralisation du contrôle-soin.
De la régulation vers le soin

Nous observons une évolution de la régulation disciplinaire vers le contrôle-soin, d'abord en termes de signalétique. La signalétique «Ojo!» (ce qui signifie « oeil » et « attention! ») a rempli un moment le métro avant la signalétique exposée ci-dessus. Elle était associée à un personnage en forme d'œil et occupait différentes surfaces, sols et murs avec des éléments signalétiques beaucoup plus grands que ceux de la campagne suivante (Figure 2). Son omniprésence et sa forme d'œil renvoyaient à un contrôle panoptique tandis que «Ojo! » peut s'interpréter comme une menace par opposition à «Cuidado » ou «Precaución » qui renvoie d'abord à une protection. Ce slogan portait déjà un ordre comportemental (regarder et se mettre en alerte) et l'annonce d'un ordre comportemental (la consigne associée). Cette signalétique qui visait aussi la diffusion d'habitudes renvoyait donc entièrementà la discipline du contrôle des corps. Le fait qu'elle ait laissé la place à la campagne « Por un metro amable » et «Cuida el Metro » nous paraît symptomatique d'une évolution d'une signalétique disciplinaire de régulation vers une autre de contrôle-soin biopolitique.
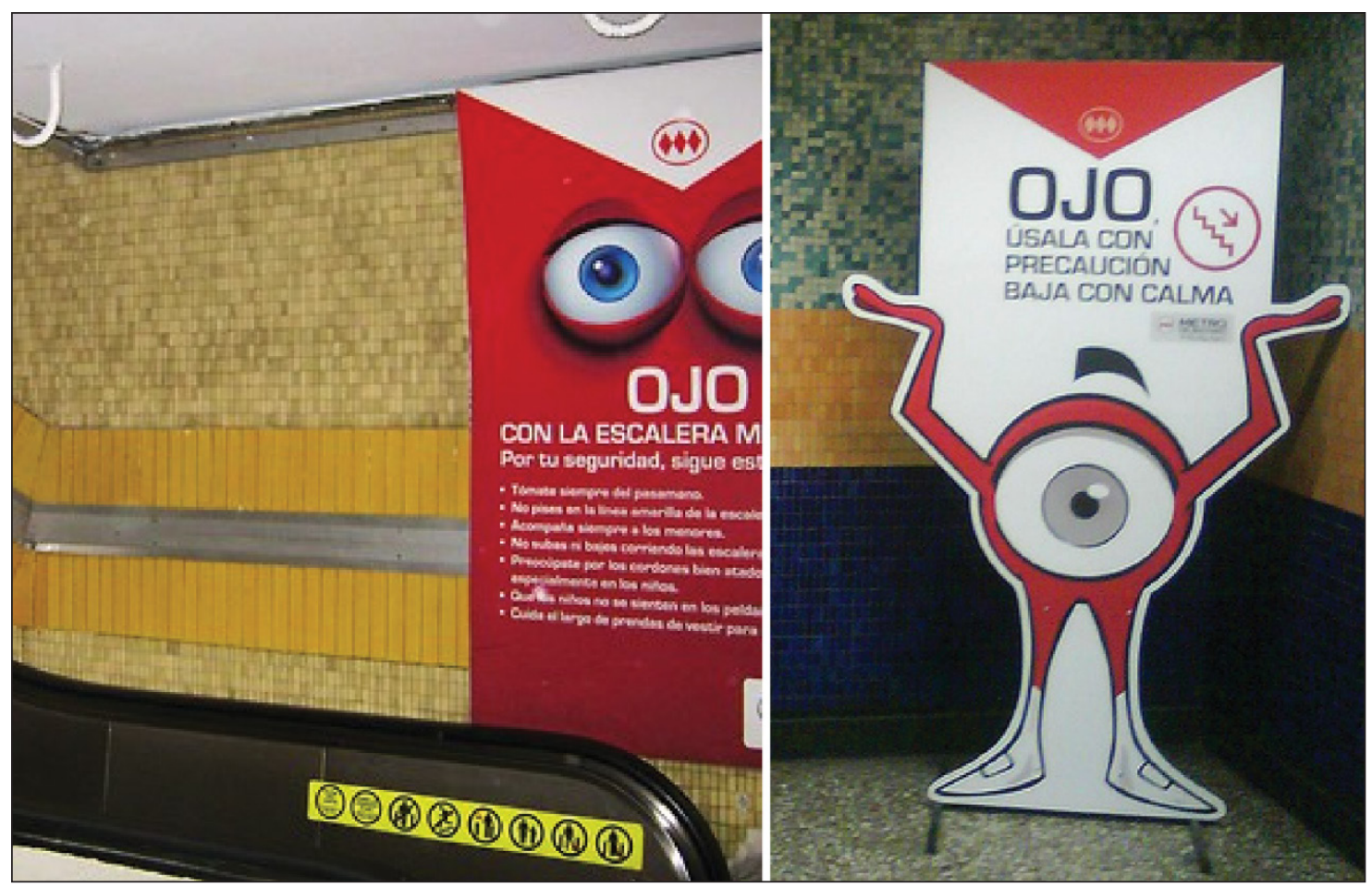

Figure 2 - Campagne « 0jo ! », métro de Santiago du Chili, 2008

Source: photo des auteurs.

urbe. Revista Brasileira de Gestão Urbana (Brazilian Journal of Urban Management), 2015 maio/ago., 7(2), 167-181 
Cette évolution nous semble apparaître aussi dans la campagne publicitaire de 2008 sur les espaces publicitaires du métro. Des usagers sont mis en scène à la fois dans leur diversité et dans leur amabilité, amabilité consubstantielle à leur identité et non à leur présence dans le métro. Ainsi un jeune punk à crête rouge déclare « Je suis aimable par nature » (« Soy amable por naturaleza ») suivi d'un slogan « ...en étant sympas on voyage mieux» (« ...con buena onda viajamos mejor ») (Figure 3). Ou un quarantenaire assuré et riant qui suscite l'intérêt de deux passagères, représentant probablement une image du séducteur macho chilien, affirme « Moi je ne dépasse jamais les bornes » («Yo nunca me paso de la raya ») suivi de la consigne « ...je franchis la ligne jaune seulement pour monter dans le métro » (« ...solo cruzo la línea amarilla para abordar el tren ») (Figure 4).

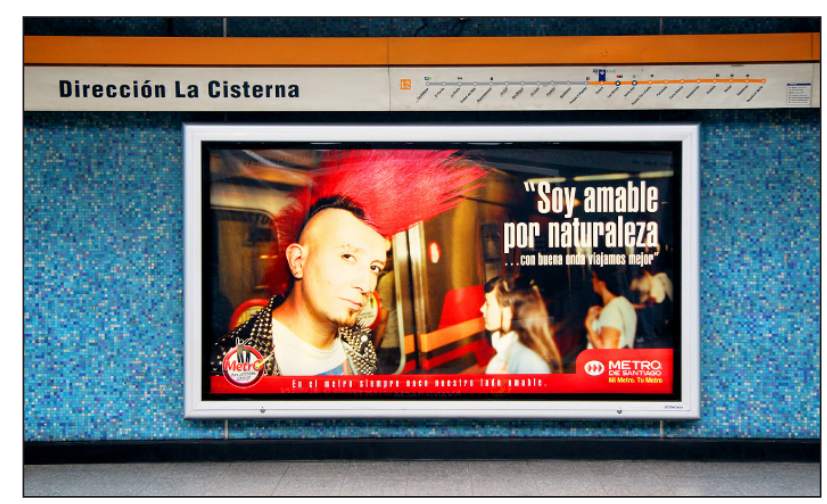

Figure 3 - Campagne d'affichage « En el metro siempre nace nuestro lado amable », métro de Santiago du Chili, 2008

Source: Photo prise le 4 septembre 2008 par Matt Hintsa (Flickr, 2008a).

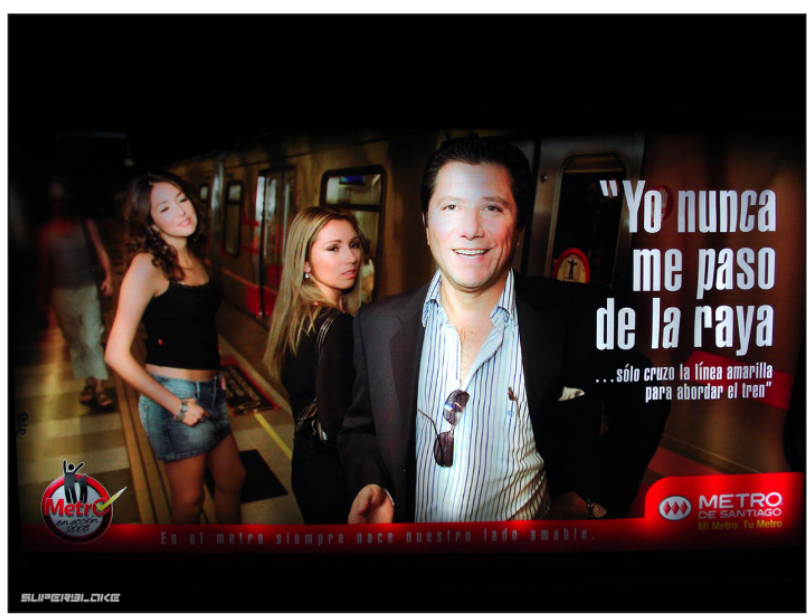

Figure 4 - Campagne d'affichage « En el metro siempre nace nuestro lado amable », métro de Santiago du Chili, 2008

Source: Photo prise le 23 juillet 2008 par Superbloke (Flickr, 2008b).
Les identités sociales ici représentées ne sont pas dénoncées mais valorisées comme éléments d'une diversité caractéristique de la population du métro et de la ville voire du pays. Par contre, ces identités sont normalisées par cette représentation publicitaire qui $\mathrm{y}$ insère le respect des autres et des règles du métro. Le bas des affiches énonce la ligne de la campagne « Dans le Métro naît toujours notre côté aimable. Métro de Santiago / Mon métro. Ton métro » ("En el Metro siempre nace nuestro lado amable. METRO DE SANTIAGO / Mi Metro. Tu Metro »). Cette ligne combine la nature intrinsèque et contingente de l'amabilité. Le caractère contingent correspond à « l'effet métro » relevé par Ureta (2013) dans la bouche des gestionnaires du métro. Elle concrétise le succès de la régulation des corps dans lesquels sont incorporées les habitudes de respect des règles. Le caractère intrinsèque renforce celui-ci qui est mis en avant dans les paroles des personnages de ces publicités. Nous notons aussi le « nous » qui renvoie à la famille et au soin, comme le slogan «Mi Metro. Tu Metro », déjà analysé.

On notera l'absence de consigne portant sur le paiement du ticket dans le métro, à la différence des bus. Pris dans son ensemble et sa diversité, le dispositif signalétique, en n'intégrant pas du tout les comportements de fraude, lui donne une autre nature, celle d'un comportement qu'il ne s'agit pas de transformer par le dressage des corps ou par l'incitation à la normalité, mais par l'interdiction de l'entrée des personnes étrangères à la population des usagers. Quant aux faiseurs de graffitis, la signalétique du métro ne s`adresse pas à eux mais aux autres usagers en les invitant à dénoncer les graffeurs. De ce fait, elle identifie aussi ces derniers comme des étrangers, distincts des usagers protégés et appelés à protéger le métro par la délation : contrôle-soin pour les usagers, menace pour les étrangers. Les campagnes du métro orientées vers le soin contrastent avec les visuels diffusés sur les bus du Transantiago sur lesquels une vieille dame ou un honnête travailleur sermonne le fraudeur, alors défini comme usager, certes récalcitrant.

L'ensemble du dispositif signalétique du métro marginalise le contrôle-menace et fait évoluer le contrôle-régulation vers un contrôle-soin, dans son format ou son contenu. Cette modalité énonce des désirs (métro aimable, propre, rapide, sûr) comme les désirs supposés des usagers de Santiago et d`ailleurs (Reigner et al., 2009; Butcher, 2011). Le contrôle-soin 
diffuse et impose ces désirs comme normalités. Celles-ci ne s'éloignent toutefois jamais beaucoup de la norme disciplinaire exigée par la régulation des corps dans le flux, ainsi renforcée par la modalité du soin.

Apparition de la régulation par réseaux sociaux sous la forme du contrôle-soin

Une extension de ce dispositif de diffusion des règles de comportement dans le métro passe par Twitter. L'usager peut suivre @metrodesantiago et être informé des changements dans le fonctionnement du métro mais aussi des consignes ordinaires. Dans les deux cas, l'information sur l'orientation des passagers à l'échelle du réseau ou à celle des machines concrètes est associée à une justification par le soin pour l'usager, soit son plaisir, son temps de trajet ou sa sécurité :

- «13h00. Ne rentre pas dans le métro après l'annonce de fermeture des portes. Tu retardes ton voyage et celui de tout le monde.» («13:00 hrs. No ingreses al tren luego del anuncio de cierre de puertas. Retrasas tu viaje y el de todos »),

- «Aide-nous à éviter les accidents, descends calmement les escaliers, un autre métro arrive déjà pour toi » («Ayúdanos a evitar accidentes, bája con calma las escaleras, otro tren ya viene porti»),

- «Ce soir nous facilitons ton arrivée au stade pour voir le Chili jouer contre l'Argentine, la station Ñuble sera desservie par tous les métros » ("Esta tarde facilitamos tu llegada al estadio para ver a Chile frente a Argentina, Estación Ñuble será común »),

- «Nous rappelons que Irarrázaval est fermée pour cause de troubles à l'extérieur. Les métros ne marqueront pas d'arrêt à cette station » ( «ecordamos que Irarrázaval está cerrada por disturbios en el exterior. Trenes no se detienen en la estación »).

Nous remarquons que l'argument selon lequel un usager qui entre après le signal sonore retarde son propre trajet est fallacieux puisquil ne retarde que les autres usagers. Il révèle néanmoins l'importance de donner à toute information une utilité pour l'usager qui la reçoit, y compris les consignes qui visent le fonctionnement général du métro. Ce formatage est compréhensible étant donné que l'inscription et la consultation de ces informations sont laissées au libre choix de l'usager. Passer de la régulation vers le soin s'impose pour ces médias optionnels qui supposent la participation volontaire de l'usager. En retour, ce contrôle-soin offre de nouvelles technologies à l'effort de diffusion du contrôle-régulation, car il s'agit toujours de consignes de régulation qui prennent la forme du soin et qui sont entourées d'informations visant le soin.

\section{Prétention au contrôle-soin des nouveaux agents de sécurité}

Absent des campagnes signalétiques de contrôle-soin, un contrôle-menace spécifique est susceptible de perdurer. Effectivement, il se poursuit par la surveillance des vigiles aux barrières d'entrée. Elle est complétée par l'action des trois autres types d'agents présents dans l'enceinte du métro, à savoir les agents de sécurité, les assistants de quai et les vendeurs de recharges téléphoniques. Même si ces deux derniers types d'agents ne sont pas censés réaliser un tel contrôle (ce dont profitent certains usagers en fraudant à côté d'eux), leurs positions et circulations créent le cadre d'une surveillance panoptique. Par contre, les agents de sécurité sont censés combiner différentes fonctions dont le contrôle-menace, alors que les autres sont spécialisés : les vigiles incarnent la menace, les assistants de quai la régulation et les vendeurs de recharges le soin sans contrôle. De plus, les agents de sécurité sont identifiés comme agents de « service au client » et sont censés aller à la rencontre des usagers afin de prévenir les conflits et rixes entres usagers ou entre usagers et assistants de quai, alors que les autres agents doivent respecter un silence total. En combinant contrôle et soin, les agents de sécurité semblent incarner le contrôle-soin : " obéis-moi car je te protège ». Le contrôle-soin s'appuie ici sur l'ambiguïté de la sécurité : celle des usagers ou celle des intérêts de Metro. Ambiguïté dialectique toute biopolitique : faire consentir à un contrôle sécuritaire car cette sécurité garantit la liberté normalisée, laquelle profite à la société de contrôle.

En fait, les fonctions ne sont pas si séparées. Les vigiles suivent une formation à « l'attention au client» («atención al cliente »), orientent en permanence les usagers, les secourent parfois et interviennent peu contre la délinquance. De même, les agents de sécurité règlent peu de conflits et font essentiellement de 
l'orientation (forme de régulation) et du contrôle du règlement. Ces agents sont les derniers à avoir envahi le métro et révèlent l'émergence du contrôle-soin. Toutefois leur déploiement suit un renforcement des agents de régulation et de menace et l'affichage de leur contrôle-soin comme " service au client » cache leur travail de régulation et de menace.

\section{Expérience du soin pour ménager les flux}

D'autres dispositifs de régulation des flux suscitent une expérience de soin susceptible d'enclencher un contre-soin envers la régulation pourtant éprouvante des flux congestionnés. Le premier de ces dispositifs est la gestion de trafic alternée (ou express) pendant les heures de pointe : une rame ne s'arrête pas à certaines stations, la suivante ne s'arrêtera qu'à celles-là et à quelques stations communes. Cette gestion fait varier l'expérience de l'usager qui sort ainsi d'une routine constante. L'épreuve voire la conscience réflexive de l'effet du dispositif favorisent alors la reconnaissance et le consentement à ses règles signalétiques très présentes.

L'automatisation de l'annonce sonore du nom de la prochaine station de métro à l'intérieur de la rame est un autre dispositif de régulation qui active le contrôle-soin. Il s'agit en effet d'une voix féminine, chargée des stéréotypes féminins du «care», à la place de la voix souvent masculine des conducteurs. L'attachée de presse de l'opérateur met en avant l'accessibilité et la sécurité pour les personnes malentendantes, mais également l'efficacité de l'ensemble du flux car les usagers se préparent à descendre avec anticipation. Cette régulation sonore inscrit dans l'inconscient le contrôle-soin matriarcal. Le soin enveloppe et sert donc ici un dispositif disciplinaire de régulation directe des corps des usagers.

\section{Intégration biopolitique du flux par lsinformation}

En plus des dispositifs qui visent explicitement l'orientation des flux, un nombre important de supports remplissent l'espace intérieur. Nous les considérons ensemble dans la mesure où ils visent tous à susciter une expérience de bien-être de l'usager. Toutefois ils doivent aussi être pris en compte dans leur influence sur les positions des corps ainsi que sur leur potentiel effet de répulsion de certaines personnes.

Il s'agit des nombreux dispositifs d'information de divertissement et de publicité, de consommation et d'ambiance. Les murs des quais sont couverts d'emplacements publicitaires. Des téléviseurs diffusent en continu la chaîne propre au métro, SubTV. Des journaux gratuits sont diffusés à l'entrée des stations de métro dans l'enceinte duquel ils sont lus et circulent. Des écrans géants sont installés sur les plateformes d'entrée de plusieurs stations. Les micros-récits du concours «Santiago en cent mots» ( «Santiago en cien palabras ») sont affichés sur les espaces publicitaires. Des œuvres d'art géantes sont exposées sur les murs des quais. Des espaces de consommation et d'accès wifi à internet ainsi que certains services culturels (MetroArte, BiblioMetro) couvrent certaines zones des stations. Tous ces éléments, ainsi que l'architecture intérieure, le mouvement des usagers et agents, ainsi qu'un travail de design intérieur construisent une ambiance, susceptible d'être rendue intelligible en termes de contrôle et de discours.

Tous les supports d'information cités ci-dessus permettent et appellent un regard-clin-d'œil, sans attention car sans attente spécifique d'information. Ils sont compatibles à la fois avec le mouvement et l'immobilité fugaces des usagers, et avec le maintien d'une partie réflexe de leur attention sur leur navigation. En conséquence, ils établissent le mouvement comme norme et s'instaurent comme éléments d'un contrôle-régulation. La disposition des espaces de consommation marchande et d'accès au wifi gratuit ainsi que les espaces culturels ne se trouvent pas sur les quais et peu dans les couloirs, réservés à la circulation aux flux dont la régulation se voit ainsi énoncée comme priorité.

Par ailleurs, si ces éléments apportent une forme de soin à l'usager, celui-ci ne semble pas associé au contrôle. Le design et l'information (instantanée) ne visent que l'oubli de l'inutilité du temps du déplacement, ou le rétablissement d'une utilité marginale par la consommation, via l'appel que constituent les publicités ou l'offre de services présente dans le métro. Ce divertissement sert surtout la régulation en rendant acceptables ses contraintes et en lui permettant d'agir directement sur les corps par une attention rendue réflexe. 


\section{Influence biopolitique sans contrôle-soin}

L'influence biopolitique de ces dispositifs ambiants se rajoute à leur effet de régulation disciplinaire, mais sans contrôle-soin. Le divertissement et l'appel à la consommation sont des modalités efficaces d'influence comme les technologies publicitaires du marketing nous le rappellent. Une usagère du métro de Santiago, Ana, observée pendant son déplacement souterrain par Bravo (2011), se surprend ainsi à répéter mécaniquement des slogans publicitaires et révèle leur influence dans ce contexte. La publicité renvoie alors a priori aux technologies biopolitiques. Toutefois, le soin associé à l'émetteur de la publicité n'appelle pas de contre-soin à proprement parler. Si l'achat est supposé libre, ce soin est une simple information. Si le soin publicitaire est assimilé à une influence non instrumentale voire subliminale de la libido, il correspond à une obligation disciplinaire, même quand il cible une sous-population. Si l'annonceur publicitaire n'exerce pas un contrôle-soin, l'opérateur Metro réalise un tel type de contrôle par l'intermédiaire de ces sources de divertissement. La profusion d'éléments censés susciter du plaisir, tout au moins divertir des désagréments du transport en commun, peut favoriser un respect général du métro.

L'opérateur de publicité du métro de Santiago est l'entreprise globale JCDecaux. Selon l'attachée de presse de l'opérateur Metro, JCDecaux revendique offrir « non seulement des supports mais aussi des idées » («no solo soportes, sino ideas »). Dans cette posture active, il aurait produit l'estimation modélisée du temps d'exposition des usagers aux visuels publicitaires dans une décomposition temporelle fine des trajets. Un découpage si fin prépare un dressage disciplinaire mais il est mis au service du contenu publicisé, en l'occurrence surtout dans les secteurs (dans l'ordre) de l'éducation, de la distribution, des sodas et des télécommunications (chiffres de JCDecaux répercutés par l'attachée de presse). La prépondérance pour l'éducation, au cœur des aspirations sociales et donc de la paix sociale, souligne l'inscription des dispositifs publicitaires dans un contrôle-soin biopolitique au sens d'une gouvernementalité de la société dans son ensemble.

De plus, l'adaptation des supports publicitaires a été pensée pour la mise en place du Transantiago, afin de garantir une nouvelle visibilité pour des fragments plus courts des déplacements à cause de la densité des autres usagers et de la proximité aux supports. Une collaboration a eu lieu entre le département de flux et le département chargé de la publicité afin d'adapter le dispositif publicitaire au nouveau dispositif régulateur des barrières. Cette adaptation cache le fait que les aménageurs de la régulation sont ainsi mis au service des concepteurs de la publicité, en leur mettant à disposition les données des futurs aménagements qui leur permettent une conception fine et dynamique donc dense et efficace des espaces publicitaires. Mais l'étroite association entre les deux départements suggère qu'elle sert aussi les régulateurs, c'est-à-dire que le design publicitaire est réciproquement mis à leur service. Les futures densité et efficacité de l'espace publicitaire serviraient donc l'efficacité de la régulation. Notre hypothèse est que la publicité est pensée comme un élément propre du design, c'est-à-dire un élément d'agrément utile pour faire accepter les aménagements contraignants de la régulation des flux. Une sous-hypothèse est que la publicité, selon les aménageurs régulateurs, divertit mieux que d'autres formats de design la masse des usagers vis-à-vis de la contrainte des aménagements de régulation : du fait de leur renouvellement, de leur actualité et de leur densité d'informations voire de la qualité de leurs formes, mais plus essentiellement dans la mesure où la publicité vise par définition à stimuler chez le plus grand nombre d'usagers les plus forts désirs de consommation. La publicité constitue en ce sens un dispositif biopolitique de stimulation de l'activité économique et vitale de la population. Finalement, l'association des départements de design publicitaire et de design régulateur met lincitation biopolitique au service de la régulation disciplinaire, et réciproquement. Cette combinaison correspond $\mathrm{au}$ " pouvoir ambiant » inscrit dans lsarchitecture d)espaces commerciaux ouverts qui contraignent le public en le séduisant (Allen, 2006). Le soin divertissant et informatif n'est pas ici directement producteur d'un contre-soin, même s'il conforte un discours massivement porté par la signalétique et contribue ainsi indirectement à l'efficacité de ce contrôle-soin là.

L'intégration de dispositifs disciplinaires dans des technologies biopolitiques s'observe également dans la proximité entre la nouvelle billettique du Transantiago et le paiement à crédit généralisé dans la société chilienne. La « carte bip » sans contact est promue pour la fluidité des circulations intermodales bien qu'il s'agisse d'un dispositif disciplinaire de 
régulation des flux. Dans les bus, elle remplace par une soumission aux machines l'éventuelle négociation tarifaire avec le chauffeur. Ce dispositif disciplinaire rapproche l'expérience du déplacement des achats par carte de crédit. Surtout, depuis peu, les recharges de la « carte bip » peuvent justement être achetées à crédit. Le contrôle disciplinaire nourrit ici aussi les incitations biopolitiques mais le contrôle-soin ne semble pas activé.

\section{Réflexions finales : forme émergente et contrôle renouvelé}

Le surgissement dans le métro de Santiago d'une foule de nouveaux usagers a impulsé le déploiement d'un grand nombre de dispositifs, essentiellement des agents et de la signalétique. Toutefois ce déploiement s'est accompagné d'une évolution des modes de contrôle des usagers. Les traces et discours laissés par les anciens dispositifs permettent d'estimer leur intensité et modalité. Le métro mobilisait essentiellement le contrôle-menace par des vigiles et barrières, la menace qui exclut l'étranger qui fraude et dégrade le métro. Une signalétique sobre et surtout un aménagement intérieur des couloirs et des rames de métro réalisaient de plus un contrôle-régulation, régulation disciplinaire des flux, moins impérieuse dans un métro peu congestionné. Les commerces et services disponibles dans l'enceinte du métro constituaient une forme de soin déconnectée d'un contrôle des usagers.

Les nombreuses campagnes de mise en place de signalétiques ont évolué vers une modalité inédite de contrôle : le contrôle-soin. Ce contrôle est résumé par le double slogan « Le métro prends soin de toi, prends soin du métro ». Conçu sur le modèle de l'autorité matriarcale, il est fondé sur un soin démesuré, typiquement vital, qui appelle un contre-soin impossible et par là, une soumission. Au-delà des slogans qui le révèlent le plus clairement, ce contrôle-soin apparaît dans toute la signalétique, dans l'information par réseaux sociaux, dans la fonction officielle de « service au client » qu'ont les nouveaux agents de sécurité, dans la communication externe de Metro, et dans l'expérience de déplacement y compris dans la profusion de services, d'informations et de divertissements. Il constitue donc une modalité inédite et généralisée.
Toutefois, les modalités de contrôle-menace et de contrôle-régulation continuent de s'exercer. Les anciens dispositifs (signalétique, aménagement, publicité, agents) maintiennent leurs modalités de contrôle ; les nouveaux agents de sécurité mettent peu en pratique le contrôle-soin ; la nouvelle signalétique mobilise le soin pour des consignes de régulation dont l'effet disciplinaire se combine avec le contrôle-soin. Les trois modalités de contrôle se combinent, s'appellent et se renforcent mutuellement. Cette synergie passe en pratique par le mélange de leurs respectives fonctions (exclure les non-usagers, réguler les flux, protéger le métro) et technologies (menace légale, dressage disciplinaire, devoir du contre-soin). D'autres technologies (imaginaire collectif, influence subliminale) et d'autres fonctions (incitation biopolitique à la consommation) s'exercent dans le métro et se mêlent aux autres. Toutefois, l'émergence du contrôle-soin (le contre-soin pour la protection du métro) et sa combinaison généralisée avec les autres technologies et fonctions de contrôle en font la forme générale des contrôles du métro.

L'émergence du contrôle-soin comme modalité spécifique et située de contrôle s'inscrit dans des processus qui dépassent l'enceinte du métro. Le contrôle-soin participe en particulier de la reconfiguration historique des modalités de gouvernement à l'échelle de la ville. De manière concomitante à la mutation des dispositifs du métro, les modalités de contrôle évoluent fortement dans les bus et gares intermodales, avec des différences notables selon les quartiers. Comparer ces modes de transport permettrait de caractériser les effets socio-spatiaux du dispositif de mobilité de Santiago pris dans son ensemble. Dans ce contexte, le contrôle-soin du métro pourrait réactualiser une ségrégation périphérique par les transports, renouveler l'agrégation graduée propre au Transantiago, ou au contraire encourager une désagrégation territoriale (Jouffe et al., 2015).

Ces processus urbains dépassent même les limites de la capitale chilienne : la sécurité « biopolitique » (Foucault, 2004b), la « société de contrôle » (Deleuze, 1990), «l'homme endetté » (Lazzarato, 2011), entre autres conceptualisations. Le contrôle-soin devrait être donc analysé comme un élément situé de modalités de gouvernement plus larges. L'incitation au surendettement dans tous les domaines de la vie, si prégnante au Chili (Pulgar, 2014), peut ainsi s'énoncer comme la forme économique du contrôle-soin : l'agrément 
d'une acquisition gratuite (le soin asymétrique) oblige à un remboursement (contre-soin) impossible à cause du montant démesuré ou des conditions usuraires du prêt. Le contre-soin viserait ici le fonctionnement néolibéral de la société qui généralise l'achat à crédit (Lazzarato, 2011). Ce contrôle-soin à l'échelle sociétale a fonctionné au vu de la grande stabilité politique du régime dit démocratique néolibéral chilien. Seuls les récents mouvements sociaux changent la donne politique, notamment sous l'action conjointe des secteurs piégés par le crédit comme les étudiants et leurs familles, ou les propriétaires surendettés (Pulgar, 2014). Des mouvements massifs ont aussi résisté en 2014 aux hausses de tarifs et à la saturation chronique du Transantiago : appel à la grève du ticket, fraude massive, piratage de billettique, dénonciations radicales du système de transport. Les métros et bus sont alors apparus à la fois comme les lieux d'un contrôle sociétal et comme les foyers d'une résistance populaire. Les usagers sous contrôle des transports souterrains sont bien des sujets gouvernés mais aussi des acteurs puissants des reconfigurations urbaines et sociales.

\section{Références}

Allen, J. (2006). Ambient power: Berlin's Postdamer Platz and the seductive logic of public spaces. Urban Studies, 43(2), 441-455. http://dx.doi.org/10.1080/00420980500416982

Amorim, M. (2015). L'effort pour rendre l'autre bête. Revue du MAUSS permanente [En ligne]. Récupéré le 10 février 2015, de http://www.journaldumauss. net/?L-effort-pour-rendre-l-autre-bete.

Bravo, R. (2011). Levantarse mas temprano no es la solucion. In Historias de viaje, Santiago se mueve. Blog. Récupéré de http://www.santiagosemueve.com

Butcher, M. (2011). Cultures of commuting: the mobile negotiation of space and subjectivity on Delhi's metro. Mobilities, 6(2), 237-254. http://dx.doi.org/10.1080/17 450101.2011 .552902

Castro, E. (2008). Biopolítica: de la soberanía al gobierno. Revista Latinoamericana de Filosofía, 34(2), 187-205.

Cova, F. (2010). Pourquoi se soucier du Care? Une recension de « Care, justice et dépendance : introduction aux théories du Care » de Marie Garrau et Alice Le Goff. Nonfiction [En ligne]. Récupéré le 10 mai 2012, de http://nonfiction.fr.

Deleuze, G. (1990). Post-scriptum sur les sociétés de contrôle, in Pourparlers 1972 - 1990. Paris: Les Éditions de Minuit.

Díaz, G., Gómez-Lobo, A., \& Velasco, A. (2006). Micros en Santiago: de enemigo público a servicio público. In A. Galetovic, \& I. Poduje, Dónde estamos y hacia dónde vamos (p. 425-460). Santiago: Centro de Estudios Públicos.

Flickr. (2008a.). Dirección La Cisterna. Récupéré le 2 décembre 2014, de http://www.flickr.com/photos/ matt_hintsa/2837832368

Flickr. (2008b.). Récupéré le 2 décembre 2014, de http:// www.flickr.com/photos/superbloke/2697304784

Foucault, M. (1975). Surveiller et punir. Naissance de la prison. Paris: Gallimard.

Foucault, M. (2004a). Sécurité, territoire, population: cours au collège de France, 1977-1978. Paris : Gallimard; Seuil.

Foucault, M. (2004b). Naissance de la biopolitique: cours au collège de France, 1978-1979. Paris : Gallimard; Seuil.

Jouffe, Y. (2015). La moyenización por la movilidad urbana: estudio de caso en un barrio popular de Santiago de Chile. In E. Zenteno, \& A. Sehtman (Eds.), Latinoamérica urbana: una mirada sobre la transformación de las ciudades, México: PUEC-UNAM.

Jouffe, Y., Lazo, A., \& Carvajal, D. (2015). Le néolibéralisme en movement : les trois constellations de mobilité de Santiago du Chili. In F. Gaudichaud \& A. Faure (Eds.), Le Chili actuel. Gouverner et résister dans une société néolibérale, Rennes: PUR.

Lazo, A. (2008). Transporte, movilidad y exclusión. El caso de Transantiago en Chile. In Diez años de cambios en el Mundo, en la Geografía y en las Ciencias Sociales, 19992008. Actas del X Coloquio Internacional de Geocrítica. Universidad de Barcelona. Récupéré de http://www.ub.es/ geocrit/-xcol/64.htm

Lazzarato, M. (2011). La fabrique de l'homme endetté, essai sur la condition néolibérale. Paris: Éditions Amsterdam.

Márquez, F. (2012). Santiago de Chile: ciudad propia, ciudad bárbara. Bitacora Urbano-Territorial, 20(1), 21-30.

Ortiz Osés, A. (2001). Mitologías culturales. In B. Solares (Ed.), Los lenguajes del símbolo. Barcelona: Anthropos. 
Pétonnet, C. (1982). L'observation flottante. L'exemple d'un cimetière parisien. L'Homme, 22(4), 37-47. http:// dx.doi.org/10.3406/hom.1982.368323

Pulgar, C. (2014). Quand la justice spatiale fait trembler la ville néolibérale. Le double mouvement tellurique et social dans le Chili d'après le tremblement de terre du 27 février 2010. Justice Spatiale, 5. Récupéré de www.jssj.org

Reigner, H., Hernandez, F., \& Brenac, T. (2009). Circuler dans la ville sûre et durable: des politiques publiques contemporaines ambiguës, consensuelles et insoutenables.
Métropoles [En ligne], 5. Récupéré le 11 janvier 2015, de http://metropoles.revues.org/3808

Ureta, S. (2013). Waiting for the Barbarians: disciplinary devices on Metro de Santiago. Organization, 20(4), 596-614. http://dx.doi.org/10.1177/1350508412452623

Velasco Maillo, H., \& Díaz de Rada, A. (1997). La lógica de la investigación etnográfica. Madrid: Editorial Trotta.

Reçu: Déc. 10, 2014

Approuvé: Févr. 13, 2015 